\title{
Review
}

\section{Regular Exercise as a Secondary Practical Treatment for Nonalcoholic Fatty Liver Disease}

\author{
Sechang Oh',2, Goeun $\mathrm{Han}^{3}$, Bokun Kim4, Junichi Shoda ${ }^{1,2^{*}}$ \\ 'The Center of Sports Medicine and Health Sciences, Tsukuba University Hospital, Tsukuba, Ibaraki 305-8576, Japan \\ ${ }^{2}$ Faculty of Medicine, University of Tsukuba, Tsukuba, Ibaraki, 305-8575, Japan \\ ${ }^{3}$ Graduate School of Comprehensive Human Sciences, University of Tsukuba, Tsukuba, Ibaraki 305-8575, Japan \\ ${ }^{4}$ Foundation for Industry-Academy Cooperation, Dong-A University, Pusan 49315, Republic of Korea.
}

\section{Article Information \\ History: \\ Received: January 11, 2018 \\ Accepted: February 12, 2018 \\ Published: March 07, 2018}

\section{Keywords:}

Exercise

Inflammation

Insulin resistance

Liver function tests

Liver steatosis

\begin{abstract}
A novel notion has arisen in clinical medicine: non-alcoholic fatty liver disease (NAFLD), representing a spectrum of conditions ranging from asymptomatic simple steatosis to steatohepatitis and cirrhosis. Even though there is a pressing need to treat this disease, the treatment options are limited. Previous studies have confirmed that weight loss via lifestyle management has proven to be successful. This is now accepted as the first-line therapy. In those studies, improvements of liver function and hepatic steatosis were noted when weight loss of around $3 \%$ to $10 \%$ of body weight was achieved. However, optimum weight loss and its management are difficult to accomplish. Subsequent studies showed that when weight loss was achieved independent of exercise, additional beneficial effects were observed in addition to weight loss. Exercise with a diet regimen brought even more benefits. Because of this substantial evidence, exercise should be proposed as another strategy for NAFLD treatment, regardless of weight loss. This review was aimed at providing a succinct, comprehensive overview of the diagnosis and management of NAFLD, highlighting the need for routinization of the role of exercise in NAFLD management, and at elucidating the mechanisms behind the benefits of exercise, with relevant studies.
\end{abstract}

\section{INTRODUCTION}

Recently, a novel notion has arisen in clinical medicine: nonalcoholic fatty liver disease (NAFLD), representing a spectrum of conditions ranging from asymptomatic simple steatosis to steatohepatitis and cirrhosis [1]. NAFLD has come to be one of the most frequently encountered conditions in daily clinical practice, recognized as a serious and growing clinical problem since the 1980s [2]. This sudden change is suspected to result primarily from environmental factors, including sedentary behavior and overeating in our current affluent society. NAFLD is known to be strongly associated with obesity and its sustaining conditions, leading to an increased risk of cardiovascular disease. It is correlated with all-cause mortality

*Correspondence: Junichi Shoda, MD, PhD, A.G.A.F., Faculty of Medicine, University of Tsukuba, 1-1-1 Tennodai, Tsukuba, Ibaraki 305-8575, Japan.

E-mail address: shodaj@md.tsukuba.ac.jp in both Western and Asian populations [3-5]. An epidemiological study estimated regional NAFLD prevalence in Japan to be $29.7 \%$ (41.0\% for males and $17.7 \%$ females) [6]. Among these, the prevalence of nonalcoholic steatohepatitis (NASH) was estimated to be about $2.7 \%$, according to the BAAT index (calculated using BMI, age, ALT, and triglyceride) $\geq 3$ [6].

Despite the high prevalence of NAFLD, treatment options are limited. Currently, there is an overall paucity of evidence for available pharmacologically- and surgically-proven benefits for treatment of NAFLD. These approaches may be exclusive to certain patients with NAFLD because they remain too expensive and risky to be applied to the general population of patients with NAFLD. By contrast, weight loss via lifestyle management has been confirmed as a first-line therapy through a growing body of evidence for the treatment of NAFLD. However, lifestyle management generally results in only modest weight loss. It is very difficult for patients to achieve and maintain an optimum range of body weight. Therefore, strategies that efficiently improve NAFLD in the absence of weight loss are of major practical significance. terms of the Creative Commons Attribution License (http://creativecommons.org/licenses/by/4.0/), which permits unrestricted use, distribution, and reproduction in any medium, provided the original work is properly cited. 
Exercise may potentially be an option to help manage the condition. Therefore, we need to establish the role of exercise as a practical secondary strategy for NAFLD, irrespective of weight loss.

In this review, we provide an opportunity to explain every aspect of NAFLD concisely yet comprehensively, especially with respect to the diagnosis and management of NAFLD. We emphasized the importance of investigating the role of exercise in NAFLD management, using relevant studies to illuminate the mechanisms underlying the benefits of exercise.

\section{Definition of NAFLD}

NAFLD includes a wide spectrum of several clinical conditions in liver disease, including asymptomatic simple steatosis, steatohepatitis, advanced fibrosis, cirrhosis, hepatocellular carcinoma, hepatoma, and liver cancer. In NAFLD, these are associated with an elevated liver fat content that is not due to excessive alcohol use (over $10 \mathrm{~g} /$ day for females, over $20 \mathrm{~g} /$ day for males). A diagnosis of NAFLD is made when screening shows at least $5 \%$ of the liver's weight top comprise lipids [7].

Not every patient showing simple steatosis reaches the subsequent cirrhotic stage; however, some patients can progress to such an advanced stage. Typically, cirrhosis is associated with a number of complications, including ascites (an accumulation of fluid in the peritoneal cavity), hepatic encephalopathy, and hepatic coma (occurrence of confusion, altered level of consciousness, and coma as a result of liver dysfunction), variceal hemorrhage (loss of blood in the gastrointestinal tract, from the pharynx to the rectum) and hepatorenal syndrome (rapid deterioration in kidney function in patients with cirrhosis or fulminant liver dysfunction) associated with a very poor prognosis [8].

\section{Progression of NAFLD}

At first, the accumulation of lipids takes place in the liver, where it is initially called benign-asymptomatic steatosis. However, when oxidative and inflammatory conditions accumulate, this stage can progress further to steatohepatitis, with the formation of scar tissue, progressive fibrosis. The struc- tural alterations broaden because of degeneration in hepatic function, leading to cirrhosis [2] (Figure 1).

\section{a. Hepatic steatosis}

The accumulation of hepatic lipids in the first stage of NAFLD is a function of four sources - circulating non-esterified fatty acids (FFA) from dietary intake and adipose tissue (mainly visceral fat), hepatic $\beta$-oxidation, de novo lipogenesis, and very low density lipoprotein (VLDL) synthesis, from both export and clearance [9].

Adipose-derived FFAs are dominant contributors to liver fat accumulation, accounting for $60 \%$ of liver fat in obese individuals with NAFLD [10], with de novo lipogenesis and dietary-derived FFAs accounting for an estimated 26\% and $15 \%$ of hepatic lipids content, respectively. Progressive insulin resistance in adipose and muscle tissues is thought to play a primary role in the increased levels of FFA. The insulin resistance in adipose tissue manifests as a decrease in the beneficial function of lipolysis suppression through insulin signaling, which in turn leads to an increased efflux of FFA [11]. This hyperinsulinemia induces activation of transcriptional regulator lipogenic factors, including sterol regulatory element binding protein-1c (SREBP1c), fatty-acid synthase, carbohydrate responsive element-binding protein, and peroxisome proliferator-activated receptor $\gamma$ [12], as well as inhibition of $\beta$-oxidation and excretion of FFA [13], all leading to a vicious cycle [14].

\section{b. NASH}

Asymptomatic steatosis in the liver is thought to have a benign clinical course in most NAFLD patients [5]. However, $\mathrm{NASH}$ represents the stage when asymptomatic steatosis begins to show hepatic oxidative/inflammatory changes [2]. In the pathogenesis of NASH, these processes are based on a "traditional two-hit hypothesis" [15]. The 'first hit', hepatic lipid accumulation, is most susceptible to damage, mediated by the 'second hit', including endogenous compounds in liver cells and environmental toxins, leading to $\mathrm{NASH}$, fibrosis and cirrhosis, i.e., inflammatory cytokines, adipokine imbalance, glucocorticoids, gut-derived endotoxin, bacterial overgrowth, endoplasmic reticulum stress, mitochondrial dysfunction,



Figure 1. The spectrum of NAFLD. 
and oxidative stress $[3,16]$.

However, the "modified two-hit hypothesis" [15] recognizes that liver fat directly causes the liver damage that results in NASH. This modified hypothesis provides substantial evidence that overproduction of FFA as a result of overeating, obesity, and insulin resistance can directly induce toxicity by increasing levels of oxidative stress (lipid peroxidation) and by activation of inflammatory signals, e.g., nuclear factor- $\kappa \mathrm{B}$ (NF- $\kappa \mathrm{B})$ [17]. Therefore, patients with NASH tend to have higher levels of oxidative stress and inflammation than do patients with simple steatosis $[18,19]$.

\section{c. Fibrosis and cirrhosis}

Fibrosis, and its more advanced stage, cirrhosis, represent the final histological pathway of many types of chronic liver diseases [20]. Typically, these stages occur as a result of reparative or reactive due to asymptomatic steatosis [21]. Cirrhosis is an advanced stage of fibrosis associated with regenerative nodules. It is variable, and generally leads to irreversible loss of liver function. These stages are explained by the "third-hit hypothesis" [15], which reflects inadequate hepatocyte proliferation after onset of NASH. Cell death stimulates the generation of new hepatocytes to compensate for dead cells and to repair tissue function [22]. However, increased oxidative stress in the progression of NAFLD reduces the ability of hepatocytes to proliferate, resulting in the recruitment of other pathways of hepatic regeneration, including the expansion of hepatic progenitor cells [23]. Therefore, this damaged proliferation of hepatocyte progenitors contributes to the advancement of fibrosis in NAFLD progression as the 'third hit' [15].

\section{Clinical features of NAFLD}

Most patients with fatty liver are asymptomatic and are diagnosed only after tests show abnormal liver function [24]. When these conditions are advanced, they are associated with physical symptoms, including fatigue, malaise, diffuse pain or pain in the upper right quadrant, and appearance of hepatoma. These symptoms are extremely substantial in patients with NASH or early stages of cirrhosis. Clinical signs including ascites, variceal hemorrhage, hepatosplenomegaly, stigmata, bruising, and jaundice caused by cirrhosis may be present, but they are less frequent [18].

Metabolic pathophysiological conditions are strongly linked with the severity of NAFLD. Therefore, most patients with NAFLD show clinical features of metabolic abnormalities including type 2 diabetes mellitus, insulin resistance, obesity and hypertriglyceridemia (increased levels of triglyceride), pro-inflammatory, and adipokines [2]. It is also likely that hepatic iron accumulation (elevated serum ferritin levels) is associated with the development of NAFLD [24].

\section{Treatment for NAFLD in weight loss}

At present, weight loss is recommended as optimal management for NAFLD $[3,25]$. Weight loss with lifestyle changes consisting of reduced caloric intake and increased physical activity has shown considerable improvements in terms of liver function tests (AST -59\% and ALT -67\% [26]) and decreased liver fat content (44\% to $58 \%$ [27]) when a body weight loss of $10 \%$ is attained. It is also possible to obtain a hepatic benefit in weight loss of $<5.0 \%$ [28-30]. Most studies have suggested that a combination of dietary restriction and physical activity clearly impacts liver function tests and hepatic steatosis when a reduction of $3 \%$ to $10 \%$ of body weight is attained. However, weight loss via lifestyle management in daily life tends to be quite modest, and moreover, is quite difficult to achieve and maintain.

\section{Exercise in NAFLD}

The specific benefit of exercise in improving NAFLD has been demonstrated in interventional studies. Improvements in liver enzymes and metabolic abnormalities in obesity were independent of detectable reductions in body weight [31-34] (Figure 2). In addition, cross-sectional studies have shown that higher physical activity levels are associated with lower levels of NAFLD pathophysiology [35-37]. In addition, there was an inverse correlation between NAFLD outcomes and cardiorespiratory fitness [38-40]. However, there are presently no specific guidelines and little available information regarding the benefits of exercise in NAFLD. In particular, the mechanisms underlying the benefits of exercise are not well clarified with respect to the pathophysiology of NAFLD.

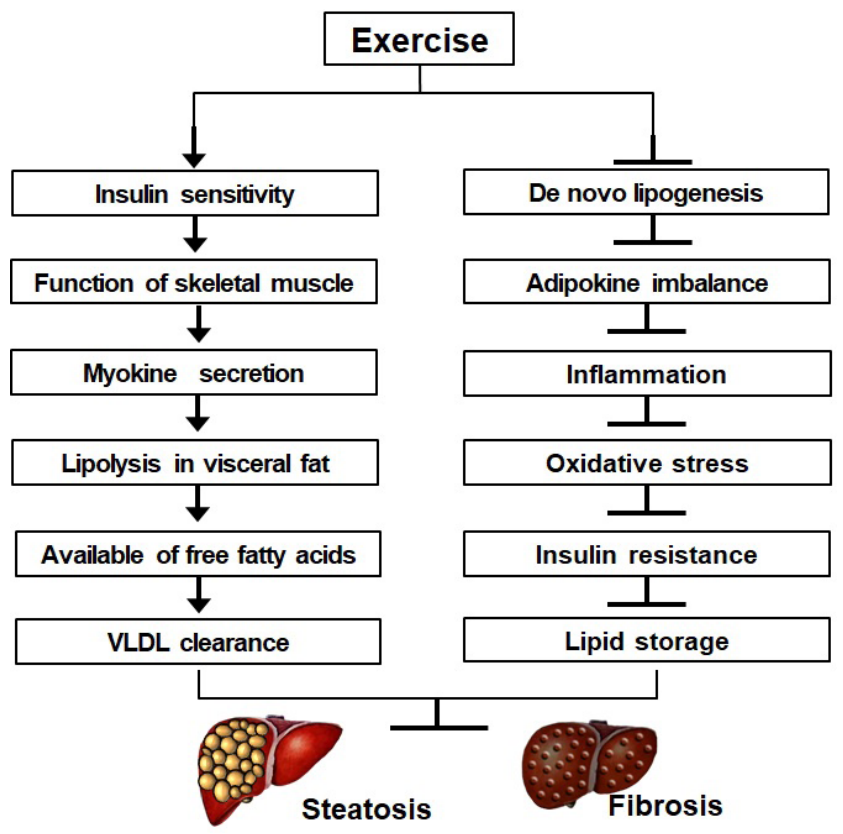

Figure 2. Schematic summary of the beneficial effects of exercise on the pathophysiological conditions associated with NAFLD, independent of weight loss. 
Mechanisms behind the effects of exercise on NAFLD pathophysiology

Exercise markedly accelerates FFA oxidation because of the increased respiration rate by stimulated skeletal muscle. FFA oxidation in exercise increases with duration and intensity, and remains in an elevated state for hours after exercise stimulus [41]. The adaptive response to exercise includes several factors that contribute to benefits, including increased mitochondrial biogenesis and capillarization for activities involved in FFA uptake, $\beta$-oxidation and fat storage in muscle [41], resulting in the excretion of FFA from the liver. Hannukainen et al. (2007) [42] reported that exercise reduced hepatic FFA uptake, and that increased FFAs were released to muscles via hepatic FFAs extraction because there was no observation of a fall in portal vein blood flow during exercise. In addition, there is likely a cumulative effect of the benefits of exercise for the liver. This contributes to the reduction of subcutaneous and visceral adiposity over time [43], and consequently reduces the accumulation of FFAs in the liver.

The feasible benefits of regular exercise also suggest the significance of improvements in the function of adipose tissue, regardless of weight loss, including modifications in insulin sensitivity and adipokine imbalance [9]. Such insulin sensitivity-induced regular exercise in adipose tissue suppresses increased lipolysis with impaired insulin action The level of insulin resistance in adipose tissue is correlated with hepatic lipid levels in patients with NAFLD [43]. By ameliorating insulin resistance through regular exercise, lower FFA levels in the patients' circulation were attained [44].

Another possible mechanism is lowering hepatic FFA uptake and increasing FFA availability. In the context of weight loss by calorie restriction, decreases in hepatic lipids paralleled the reduction of hepatic FFAs uptake, despite similar basal FFAs levels [45]. Such a result suggests that the liver is not only a passive site for adipose tissue action, but it is also an active regulator of hepatic FFA uptake. It is likely that the uptake is controlled [46] via expression of the FFA translocator or the cluster of differentiation 36 (CD 36). Exercise regulates lipid metabolism via altered expression of CD 36 [47]. Recent studies have shown that exercise increased VLDL secretion and clearance [48] in the liver, but the contribution of VLDL lipids-derived FFAs is inconsequential compared with that of adipocyte- and intramyocellular lipids-derived lipolysis [41]. Exercise may control hepatic lipids by directly increasing $\beta$-oxidation and inhibiting lipogenesis in the liver [9].

Low cardiorespiratory capacity increases the level of sterol regulatory element binding protein 1c (SREBP-1c), a lipogenic transcription factor known to regulate genes promoting triglyceride production. According to Thyfault et al. (2009) [49], when compared with sedentary rats, rats with a high level of fitness had lower visceral adiposity and less insulin resistance due to SREBP-1c inhibition. The mechanism suggested by this group was that peroxisome proliferator-activated receptor- $\delta$, involved in the regulation of hepatic lipogenesis, acts by suppressing SREBP-1c activity and by inducing the expression of insulin-induced gene-1 [50]. The signal for these adaptations is strongly related to an increase in adenosine monophosphate-activated protein kinase (AMPK), which is activated by the increased exercise stimulus. Up-regulation of AMPK was shown to reduce malonyl-coenzyme A, which regulates FFA entry and $\beta$-oxidation in mitochondria, as well as lipogenesis and insulin resistance. These effects are controlled by adiponectin, which activates AMPK in the liver. Adiponectin levels have been shown to increase as a result of exercise, demonstrating an independent benefit of weight loss.

Recently, some investigators have reported that exercise may modulate Nrf2 (nuclear factor-E2-related factor 2) signaling in rat kidney [51], mouse heart [52], and human skeletal muscle [53]. The transcription factor Nrf2 is a master regulator of the antioxidant responsive element-driven cellular defense system against oxidative and electrophilic stresses. Activation of the Nrf2 regulatory pathway has been shown to improve pathological factors. These include inhibition of lipid accumulation in the liver and inflammation by the nuclear factor NF-kappa B pathway, reduction of oxidative stress by superoxide dismutase and catalase activities, and reduction of hepatic fibrosis by inhibition of transforming growth factor beta 1 [54]. Therefore, Nrf2 activation through exercise stimulus might be the key factor for the therapeutic efficacy of exercise in NAFLD. However, the mechanisms of Nrf2 activation and its downstream transcriptional regulation of antioxidants have not been clearly elucidated to date.

\section{CONCLUSIONS}

It should be emphasized that the general public have misconceptions about the primary role of exercise. Exercise does not function simply as a weight control therapy, but it can potentially reverse disease progression, or at least lessen the risk of complications. In the fields of clinical medicine and physiology, the benefits of exercise are apparent. These benefits include ameliorated insulin resistance, improved lipid content and function, decreased inflammation and oxidative stress, improved health at the organ level, and prevention of other complications beyond weight reduction [9].

Body weight reduction should be the ultimate target of NAFLD treatment. However, the advantages and accrued effects of exercise in combination with a diet regimen should be scrutinized as the secondary treatment option for NAFLD [55-58]. Moreover, there is an increasing need to comprehend the molecular mechanism modulations that accompany direct and indirect facilitators induced by the exercise benefits for NAFLD. The management and initialization of exercise should be further investigated, including exercise dose, intervention, and the beneficial impacts of well-programmed exercise. Further reports on means of promoting exercise will aid in the establishment of time- and quality-efficient regimens that will not only improve overall health of patients, but patient commitment as well.

We suggest that patients with NAFLD should be encouraged to pursue the beneficial effects that can be acquired through exercise. Treatment of NAFLD should include evaluating the levels of cardiac function and physical capacity, as 
well as the setting of goals based on the attainment of sustainable exercise habits. We strongly recommend 30-60 minutes or more of moderate- to vigorous-intensity aerobic training on a continuing basis at least 5 days per week [59]. Moreover, moderate- to vigorous-intensity resistance training 3 days per week might also be required, in particular for enhancing insulin sensitivity [60]. Of course, the suggested exercise doses and modalities cannot be established as a starting point. These are intended as an optimal goal following a gradual increase in exercise [61]. It is not an easy task for most patients with NAFLD, but we strongly believe that such exercise habituation will provide definitive benefits related to hepatic steatosis and its associated pathological conditions, even in the absence of weight loss.

\section{ACKNOWLEDGEMENTS}

We thank Robert Lindeman, $\mathrm{MD}, \mathrm{PhD}$, from Edanz Group (www.edanzediting.com) for editing a draft of this manuscript.

\section{Funding}

This work was supported in part by Grants-in-Aid for Scientific Research from the Ministry of Education, Culture, Sports, Science and Technology of Japan (No. 16H03255, 16K15188, 17H02174, 17K19887).

\section{Conflicts of interest}

The authors declare no conflict of interest.

\section{ORCID}

Sechang Oh (http://orcid.org/0000-0002-7457-9305)

\section{REFERENCES}

1. Pagadala MR, McCullough AJ. Non-alcoholic fatty liver disease and obesity: not all about body mass index. Am J Gastroenterol. 2012; 107(12):1859-1861.

2. Shyangdan D, Clar C, Ghouri N, et al. Insulin sensitisers in the treatment of non-alcoholic fatty liver disease: a systematic review. Health Technol Assess. 2011; 15(38):1110.

3. Day CP. Non-alcoholic fatty liver disease: current concepts and management strategies. Clin Med. 2006; 6(1):19-25.

4. Boffetta P, McLerran D, Chen Y, et al. Body mass index and diabetes in Asia: a cross-sectional pooled analysis of 900,000 individuals in the Asia cohort consortium. PloS One. 2011; 6(6):e19930.

5. Matteoni CA, Younossi ZM, Gramlich T, Boparai N, Liu YC, McCullough AJ. Nonalcoholic fatty liver disease: a spectrum of clinical and pathological severity. Gastroenterology. 1999; 116(6):1413-1419.

6. Eguchi Y, Hyogo H, Ono M, et al. Prevalence and associated metabolic factors of nonalcoholic fatty liver disease in the general population from 2009 to 2010 in Japan: a multicenter large retrospective study. J Gastroenterol. 2012; 47(5):586-595.

7. Chitturi S, Farrell GC, Hashimoto E, Saibara T, Lau GK,
Sollano JD. Non-alcoholic fatty liver disease in the AsiaPacific region: Definitions and overview of proposed guidelines. J Gastroenterol Hepatol. 2007; 22(6):778-787.

8. Propst A, Propst T, Zangerl G, Ofner D, Judmaier G, Vogel W. Prognosis and life expectancy in chronic liver disease. Dig Dis Sci. 1995; 40(8):1805-1815.

9. Johnson NA, George J. Fitness versus fatness: moving beyond weight loss in nonalcoholic fatty liver disease. Hepatology. 2010; 52(1):370-381.

10. Preiss D, Sattar N. Non-alcoholic fatty liver disease: an overview of prevalence, diagnosis, pathogenesis and treatment considerations. Clin Sci. 2008; 115(5):141-150.

11. Korenblat KM, Fabbrini E, Mohammed BS, Klein S. Liver, muscle, and adipose tissue insulin action is directly related to intrahepatic triglyceride content in obese subjects. Gastroenterology. 2008; 134(5):1369-1375.

12. Adams LA, Angulo P, Lindor KD. Nonalcoholic fatty liver disease. CMAJ. 2005; 172(7):899-905.

13. Postic C, Girard J. Contribution of de novo fatty acid synthesis to hepatic steatosis and insulin resistance: lessons from genetically engineered mice. J Clin Invest. 2008; 118(3):829-838.

14. Fabbrini E, Sullivan S, Klein S. Obesity and nonalcoholic fatty liver disease: biochemical, metabolic, and clinical implications. Hepatology. 2010; 51(2):679-689.

15. Dowman JK, Tomlinson J, Newsome P. Pathogenesis of non-alcoholic fatty liver disease. QJM. 2009; 103(2):7183.

16. Day CP, James OF. Steatohepatitis: a tale of two "hits"?. 1998; 114(14):842-845.

17. Feldstein AE, Werneburg NW, Canbay A, et al. Free fatty acids promote hepatic lipotoxicity by stimulating TNF- $\alpha$ expression via a lysosomal pathway. Hepatology. 2004; 40(1):185-194.

18. Sanyal AJ. AGA technical review on nonalcoholic fatty liver disease. Gastroenterology. 2002; 123(5):1705-1725.

19. VIDELA LA, Rodrigo R, Orellana $M$, et al. Oxidative stress-related parameters in the liver of non-alcoholic fatty liver disease patients. Clin Sci. 2004; 106(3):261-268.

20. Farrell GC, Larter CZ. Nonalcoholic fatty liver disease: from steatosis to cirrhosis. Hepatology. 2006; 43(S1):S99-S112.

21. Day CP. Natural history of NAFLD: remarkably benign in the absence of cirrhosis. Gastroenterology. 2005; 129(1):375-378.

22. Jou J, Choi SS, Diehl AM. Mechanisms of disease progression in nonalcoholic fatty liver disease. Semin Liver Dis. 2008; 28(04):370-379.

23. Roskams T, Yang SQ, Koteish A, et al. Oxidative stress and oval cell accumulation in mice and humans with alcoholic and nonalcoholic fatty liver disease. Am J Pathol. 2003; 163(4):1301-1311.

24. Alba L, Lindor K. Non-alcoholic fatty liver disease. Aliment Pharmacol Ther. 2003; 17(8):977-986.

25. Harrison SA, Day CP. Benefits of lifestyle modification in NAFLD. Gut. 2007; 56(12):1760-1769.

26. Ueno T, Sugawara H, Sujaku K, et al. Therapeutic effects 
of restricted diet and exercise in obese patients with fatty liver. J Hepatol. 1997; 27(1):103-107.

27. Larson-Meyer DE, Heilbronn LK, Redman LM, et al. Effect of calorie restriction with or without exercise on insulin sensitivity, $\beta$-cell function, fat cell size, and ectopic lipid in overweight subjects. Diabetes Care. 2006; 29(6):1337-1344.

28. Huang MA, Greenson JK, Chao C, et al. One-year intense nutritional counseling results in histological improvement in patients with non-alcoholic steatohepatitis: a pilot study. Am J Gastroenterol. 2005; 100(5):1072-1081.

29. Kantartzis K, Thamer C, Peter A, et al. High cardiorespiratory fitness is an independent predictor of the reduction in liver fat during a lifestyle intervention in non-alcoholic fatty liver disease. Gut. 2009; 58(9):1281-1288.

30. Osland EJ, Powell EE, Banks M, Jonsson JR, Hickman IJ. Obesity management in liver clinics: translation of research into clinical practice. J Gastroenterol Hepatol. 2007; 22(4):504-509.

31. Johnson NA, Sachinwalla T, Walton DW, et al. Aerobic exercise training reduces hepatic and visceral lipids in obese individuals without weight loss. Hepatology. 2009; 50(4):1105-1112.

32. Hallsworth K, Fattakhova G, Hollingsworth KG, et al. Resistance exercise reduces liver fat and its mediators in non-alcoholic fatty liver disease independent of weight loss. Gut. 2011, 60(9): 1278-1283.

33. Shojaee-Moradie F, Baynes K, Pentecost C, et al. Exercise training reduces fatty acid availability and improves the insulin sensitivity of glucose metabolism. Diabetologia. 2007; 50(2):404-413.

34. St George A, Bauman A, Johnston A, Farrell G, Chey T, George J. Independent effects of physical activity in patients with nonalcoholic fatty liver disease. Hepatology. 2009; 50(1):68-76.

35. Hsieh SD, Yoshinaga H, Muto T, Sakurai Y. Regular physical activity and coronary risk factors in Japanese men. Circulation. 1998; 97(7):661-665.

36. Perseghin G, Lattuada G, De Cobelli F, et al. Habitual physical activity is associated with intrahepatic fat content in humans. Diabetes Care. 2007; 30(3):683-688.

37. Tiikkainen $M$, Tamminen M, Häkkinen AM, et al. Liver-fat accumulation and insulin resistance in obese women with previous gestational diabetes. Obesity. 2002; 10(9):859-867.

38. Church TS, Kuk JL, Ross R, Priest EL, Biltoff E, Blair SN. Association of cardiorespiratory fitness, body mass index, and waist circumference to nonalcoholic fatty liver disease. Gastroenterology. 2006; 130(7):2023-2030.

39. McMillan KP, Kuk JL, Church TS, Blair SN, Ross R. Independent associations between liver fat, visceral adipose tissue, and metabolic risk factors in men. Appl Physiol Nutr Metab. 2007; 32(2):265-272.

40. Nguyen-Duy T-B, Nichaman MZ, Church TS, Blair SN, Ross R. Visceral fat and liver fat are independent predictors of metabolic risk factors in men. Am J Physiol Endocrinol Metab. 2003; 284(6):E1065-E1071.
41. Johnson NA, Stannard SR, Thompson MW. Muscle triglyceride and glycogen in endurance exercise. Sports Med. 2004; 34(3):151-164.

42. Hannukainen JC, Nuutila P, Ronald B, et al. Increased physical activity decreases hepatic free fatty acid uptake: a study in human monozygotic twins. J Physiol. 2007; 578(1):347-358.

43. Chaston T, Dixon J. Factors associated with percent change in visceral versus subcutaneous abdominal fat during weight loss: findings from a systematic review. Int J Obes. 2008; 32(4):619-628.

44. Solomon TP, Haus JM, Marchetti CM, Stanley WC, Kirwan JP. Effects of exercise training and diet on lipid kinetics during free fatty acid-induced insulin resistance in older obese humans with impaired glucose tolerance. Am J Physiol Endocrinol Metab. 2009; 297(2):E552-E559.

45. Viljanen AP, Iozzo P, Borra R, et al. Effect of weight loss on liver free fatty acid uptake and hepatic insulin resistance. J Clin Endocrinol Metab. 2009; 94(1):50-55. 10.1210/ jc.2008-1689.

46. Ravikumar B, Carey P, Snaar J, et al. Real-time assessment of postprandial fat storage in liver and skeletal muscle in health and type 2 diabetes. Am J Physiol Endocrinol Metab. 2005; 288(4):E789-E797.

47. Linden MA, Fletcher JA, Morris EM, et al. Treating NAFLD in OLETF rats with vigorous-intensity interval exercise training. Med Sci Sports Exerc. 2015; 47(3):556.

48. Morio B, Holmbäck U, Gore D, Wolfe RR. Increased VLDL-TAG turnover during and after acute moderate-intensity exercise. Med Sci Sports Exerc. 2004; 36(5):801806.

49. Thyfault JP, Rector RS, Uptergrove GM, et al. Rats selectively bred for low aerobic capacity have reduced hepatic mitochondrial oxidative capacity and susceptibility to hepatic steatosis and injury. J Physiol. 2009; 587(8):18051816.

50. Qin X, Xie X, Fan Y, et al. Peroxisome proliferator-activated receptor-delta induces insulin-induced gene-1 and suppresses hepatic lipogenesis in obese diabetic mice. Hepatology. 2008; 48(2):432-441.

51. Asghar M, George L, Lokhandwala MF. Exercise decreases oxidative stress and inflammation and restores renal dopamine D1 receptor function in old rats. Am J Physiol Renal Physiol. 2007; 293(3):F914-F919.

52. Muthusamy VR, Kannan S, Sadhaasivam K, et al. Acute exercise stress activates Nrf2/ARE signaling and promotes antioxidant mechanisms in the myocardium. Free Radic Biol Med. 2012; 52(2):366-376.

53. Safdar A, Tarnopolsky MA. Dysfunctional Nrf2-Keap1 redox signaling in skeletal muscle of the sedentary old. Free Radic Biol Med. 2010; 49(10):1487-1493.

54. Bataille A, Manautou J. Nrf2: a potential target for new therapeutics in liver disease. Clin Pharmacol Ther. 2012; 92(3):340-348.

55. Oh S, Tanaka K, Warabi E, Shoda J. Exercise reduces inflammation and oxidative stress in obesity-related liver diseases. Med Sci Sports Exerc. 2013; 45(12):2214-2222. 
56. Oh S, Tanaka K, Tsujimoto T, So R, Shida T, Shoda J. Regular exercise coupled to diet regimen accelerates reduction of hepatic steatosis and associated pathological conditions in nonalcoholic fatty liver disease. Metab Syndr Relat Disord. 2014; 12(5):290-298.

57. Oh S, Shida T, Sawai A, et al. Acceleration training for managing nonalcoholic fatty liver disease: a pilot study. Ther Clin Risk Manag 2014; 10:925-936.

58. Oh S, Maruyama T, Eguchi K, et al. Therapeutic effect of hybrid training of voluntary and electrical muscle contractions in middle-aged obese women with nonalcoholic fatty liver disease: a pilot trial. Ther Clin Risk Manag. $2015 ; 11: 371-380$.
59. Oh S, Shida T, Yamagishi K, et al. Moderate to vigorous physical activity volume is an important factor for managing nonalcoholic fatty liver disease: a retrospective study. Hepatology. 2015; 61(4):1205-1215.

60. Donnelly JE, Blair SN, Jakicic JM, Manore MM, Rankin JW, Smith BK. American College of Sports Medicine Position Stand. Appropriate physical activity intervention strategies for weight loss and prevention of weight regain for adults. Med Sci Sports Exerc. 2009; 41(2):459-471.

61. Oh S, So R, Shida T, et al. High-Intensity Aerobic Exercise Improves Both Hepatic Fat Content and Stiffness in Sedentary Obese Men with Nonalcoholic Fatty Liver Disease. Sci Rep. 2017; 7:43029. 